\title{
Research on the Rolling Motion Mode of a SWATH-USV
}

\author{
Email address: \\ 1244643919@qq.com (Qin Yuxuan) \\ *Corresponding author
}

Qin Yuxuan*, Gao Shasha, Yu Yongqiang, Tang Yang, Yang Songlin

School of Naval Architecture \& Ocean Engineering, Jiangsu University of Science and Technology, Zhenjiang, China

\section{To cite this article:}

Qin Yuxuan, Gao Shasha, Yu Yongqiang, Tang Yang, Yang Songlin. Research on the Rolling Motion Mode of a SWATH-USV. American Journal of Mechanical and Industrial Engineering. Vol. 3, No. 5, 2018, pp. 91-98. doi: 10.11648/j.ajmie.20180305.13

Received: October 28, 2018; Accepted: December 3, 2018; Published: December 11, 2018

\begin{abstract}
This paper takes a SWATH-USV as the research object, and carries out a series of static water roll attenuation tests under different drafts and different initial roll angles. In order to study the roll motion mode of the SWATH-USV, we have established three mathematical models of the roll motion mode, and written the system identification software in C\# language, based on the system identification theory and the genetic programming algorithm. Firstly, by calculating the predicted values of angular velocity under different drafts and different initial roll angles, the error between the test value and the fitted value was analyzed, and the consistency between the predicted value and the test result was found to be good, which proves the reliability of the system identification software. Secondly, through the further processing of the test data, the variation law of the torque coefficient affecting the rolling motion with the draft and the initial roll angle is preliminarily analyzed. Finally, the polynomial response surface method is applied to fit the equation containing the rolling hydrodynamic torque coefficient and the recovery moment coefficient, and the equation is found to be applicability. The analysis results and methods can provide reference for the study of the wave resistance of such USV, and provide important technical support for the subsequent optimization of the boat type.
\end{abstract}

Keywords: SWATH-USV, Ship Model Test, System Identification, Roll Motion Mode, Response Surface Fitting

\section{Introduction}

During the maritime navigation, the ship is affected by the load of the marine environment, which inevitably produces complex movements. It has strong randomness and nonlinearity, which not only affects the navigation safety of the ship, but also increases the navigational resistance of the ship and affects its Sailing performance. Therefore, it is very important to study the roll motion mode of SWATH-USV and forecast its roll motion. Model test is an effective means to predict roll motion. The system identification method based on the optimization design idea has been ideally applied in the roll motion test analysis of single ship model and three-body ship model. For the analysis method of roll motion, Ding Yong, Hu Kaiye, Qiu Minzhi et al. [1] of Harbin Engineering University used the Lyapunov characteristic index to accurately find the threshold of ship nonlinear roll motion, and analyzed the global stability of ship nonlinear roll motion. Zhang Zeguo et al [2] of Dalian Maritime University used the gray model to preprocess the roll data to weaken the nonlinear influence factors in the roll state, and designed the adaptive neural blur based on the gray model particle swarm optimization algorithm inference system. Ma Lei, Zhang Xianku [3] used the Lyapunov method and power spectrum method to verify the chaotic phenomenon of the multi-degree-of-freedom ship parameter excitation roll model, and proposed the backstepping method and the closed-loop gain forming algorithm to combine the fin stabilizer controller with the ship parameters. Incentive roll motion is controlled. Li Hui et al [4] used the wavelet analysis method to analyze the nonlinear time series of ship rolling motion for time-frequency two-domain analysis, greatly increasing the one-time foreseeing period, and implementing long-term forecasting to improve the accuracy of modeling and forecasting. Li Peiyong et al. [5] of Shanghai Jiaotong University used the energy method to calculate the triangulation roll damping coefficient from the free roll attenuation test measurement data, and analyzed the characteristics of the damping nonlinear action during the roll process, and discussed the lateral arrangement position of the 
side body, the influence of bilge keel and speed on the rolling motion of the trimaran is compared with the linear and nonlinear methods for predicting the rolling motion of the trimaran. Li Hongxia, Lu Jiang et al. [6] of Dalian University of Technology considered the influence of instantaneous wet surface of the ship and the mutual coupling between heave, roll and pitch, and proposed the numerical simulation method of the coupled motion of freedom and the roll of freedom. Based on the numerical simulation method of motion, the multi-scale method is used to find the analytical solution, and the stable domain of the trivial solution and the periodic solution are obtained. Liu Hongmei of Tianjin University [7] studied the wind as a kind of power, calculated the high-order equations about the roll angle and the encounter frequency under the combined action of transverse waves, cross winds and wind waves, and modeled the frequency of each encounter to figure out the roll angle. A. Lavrov et al. [8] used the solution of the implemented Navier-Stokes equation in Open FOAM to simulate the flow near the 2D ship part of the forced roll motion. The Lagrang-Eulerian adaptive grid scheme is applied to solve the forced motion of the ship in still water and the problem of free surface capture.

In this paper, the static water roll motion mode of the SWATH-USV model was experimentally analyzed. According to the principle of roll-and-balance dynamic balance, the mathematical models of linear and nonlinear two roll-shake motion modes were established. Determine the relevant design variables and objective functions, and adapt the C\#-based genetic algorithm and particle swarm algorithm system identification program. Through the identification program, the data of the roll test were separately identified and analyzed. It can be seen that the relative error between the test value and the forecast value is small and the degree of fitting is good, which proves the reliability of the system identification method.

\section{System Identification Method}

Roll identification equation 1:

$$
\begin{array}{r}
\ddot{\varphi}+2 N \dot{\varphi} / I_{x x}^{\prime}+W|\dot{\varphi}| \dot{\varphi} / I_{x x}^{\prime}+x \varphi^{3} / I_{x x}^{\prime}+C_{1} / I_{x x}^{\prime} \varphi^{3} \\
+C_{2} / I_{x x}^{\prime} \varphi^{2}+C_{3} / I_{x x}^{\prime} \varphi=0
\end{array}
$$

Select design variables:, $I_{x x}^{\prime}, N, W, x, C_{1}, C_{2}, C_{3}$ design variable value range:

$$
\begin{aligned}
& I_{x x}^{\prime} \in[0,0.1], N \in[0,1], W \in[0,1], x \in[0,10], \\
& h \in[0,10], C_{1} \in[0,10], C_{2} \in[0,10], C_{3} \in[0,10]
\end{aligned}
$$

Roll identification equation 2 :

$$
\ddot{\varphi}+2 N \dot{\varphi} / I_{x x}^{\prime}+W|\dot{\varphi}| \dot{\varphi} / I_{x x}^{\prime}+x \varphi^{3} / I_{x x}^{\prime}+D h \varphi / I_{x x}^{\prime}=0
$$

Select design variables: $I_{x x}^{\prime}, N, W, x, h$, design variable value range:

$$
I_{x x}^{\prime} \in[0,0.1], N \in[0,1], W \in[0,1], x \in[0,10], h \in[0,10] \text { Roll }
$$

identification equation 3 :

$$
\begin{array}{r}
\ddot{\varphi}+2 N \dot{\varphi} / I_{x x}^{\prime}+W|\dot{\varphi}| \dot{\varphi} / I_{x x}^{\prime}+x \varphi^{3} / I_{x x}^{\prime}+C_{1} / I_{x x}^{\prime}(\sin \varphi)^{3} \\
+C_{2} / I_{x x}^{\prime}(\sin \varphi)^{2}+C_{3} / I_{x x}^{\prime} \sin \varphi=0
\end{array}
$$

The above four identification models, by measuring the angle of the test $\mathrm{K}$, have the error estimation criteria at the $\mathrm{K}+1$ th time as follows:

$$
\varepsilon_{k+1}=\dot{\varphi}_{k+1}^{\prime}-\dot{\varphi}_{k+1}
$$

Where: $\dot{\varphi}_{K+1}$ is the measured value of the angular velocity of $\mathrm{K}+1$; the value obtained by the identification result.

Then the objective function is selected as shown in equation 5. The smaller the objective function value is, the better the formula fitting effect is.

$$
F(x)=\sqrt{1 / N \sum_{k=1}^{N} \varepsilon^{2}{ }_{\mathrm{K}+1}}
$$

\section{System Identification Analysis}

\subsection{Analysis of Roll Test Results}

As shown in Figure1, when the initial roll angle is $7^{\circ}$, under the three different drafts, the peak value of the roll angular velocity increases with the increase of the draft and the decrease of the roll cycle, indicating that when the ballast increases, the draught is larger and the wave resistance performance is better. The peak value of the 1.1 times draft in the observation chart is smaller than the design draft, the reason may be that the the initial roll angle is forced by artificial pressure, which had an error. Observing the black curve (0.9 times draught) in the figure, the attenuation law appeares abnormal after $10 \mathrm{~s}$. The reason may be that the wave disturbance was caused by human factors, which made the rolling motion worse.

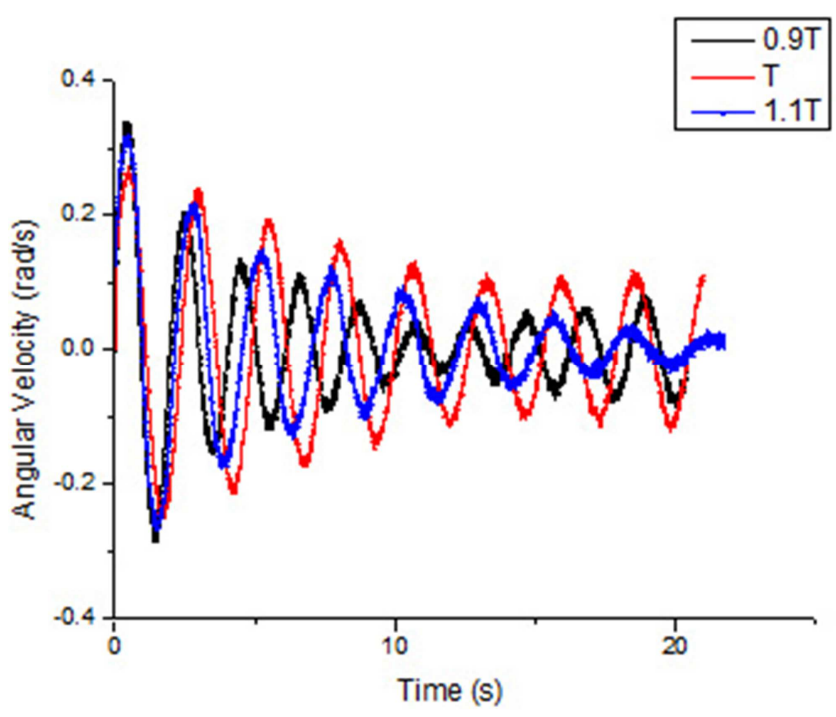

Figure 1. Rolling angular velocity decay curve for different drafts and roll initial angles of $7^{\circ}$. 


\subsection{Rolling Identification Calculation Scheme}

Three identification equations were selected in turn to perform the identification calculation analysis.

Table 1. Objective function values of four kinds of identification mathematical models.

\begin{tabular}{lllll}
\hline Objective function value & & Formula1 & Formula2 & Formula3 \\
\hline & $0.9 \mathrm{~T}$ & 0.0074 & 0.0080 & 0.0081 \\
Initial roll angle $7^{\circ}$ & $\mathrm{T}$ & 0.00757 & 0.0409 & 0.0075 \\
& $1.1 \mathrm{~T}$ & 0.0408 & 0.0095 & 0.0408 \\
& $0.9 \mathrm{~T}$ & 0.0073 & 0.0104 & 0.0073 \\
Initial roll angle $15^{\circ}$ & $\mathrm{T}$ & 0.0178 & 0.0189 & 0.0165 \\
& $1.1 \mathrm{~T}$ & 0.0210 & 0.0266 & 0.0182 \\
Initial roll angle $20^{\circ}$ & $0.9 \mathrm{~T}$ & 0.0256 & 0.0114 & 0.0256 \\
\hline
\end{tabular}

It can be seen from the figure2. that the test angular velocity and the identification angular velocity have a good fitting effect, which can prove the reliability of the identification software compiled in this paper. Therefore, the identification software can predict the roll angular velocity at the next moment.
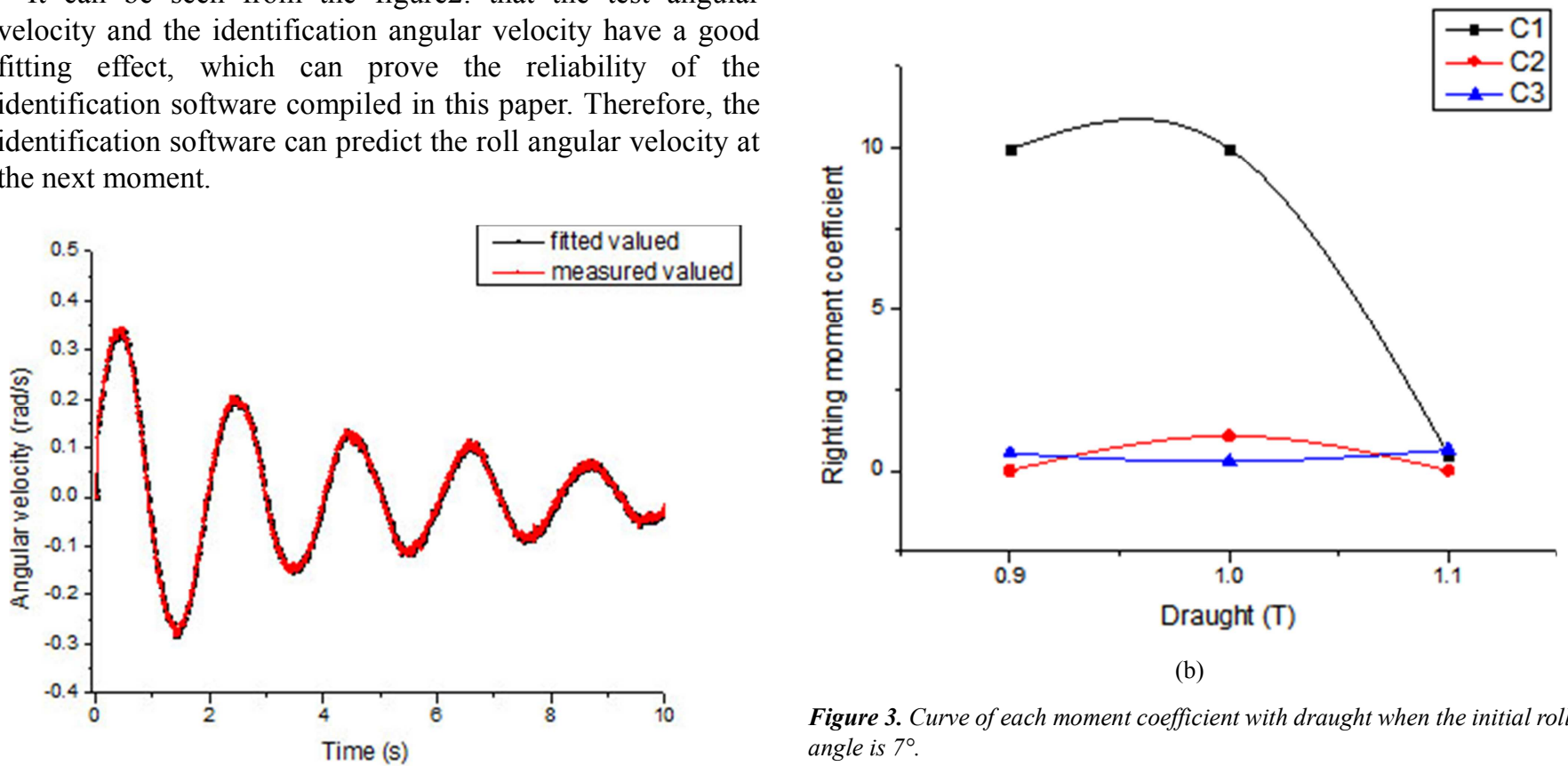

(b)

Figure 3. Curve of each moment coefficient with draught when the initial roll angle is $7^{\circ}$.

Figure 2. Test angular velocity and identification angular velocity fitting curve.
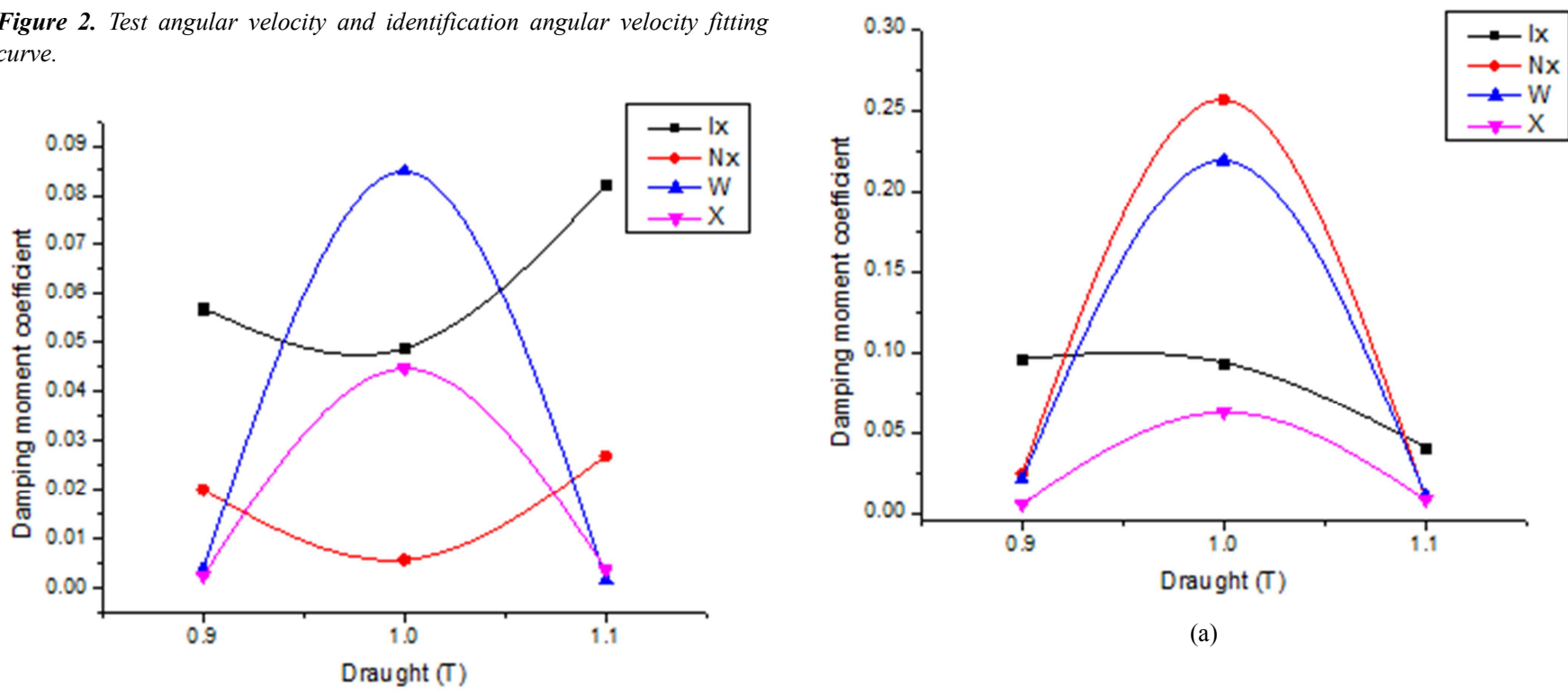

(a)

(a) 


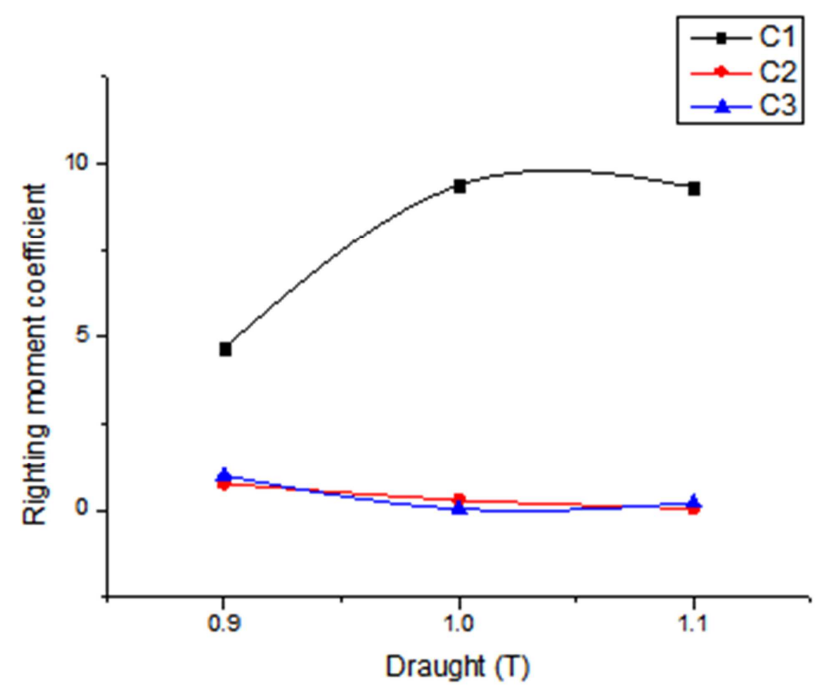

(b)

Figure 4. Curve of each moment coefficient with draught when the initial roll angle is $15^{\circ}$.

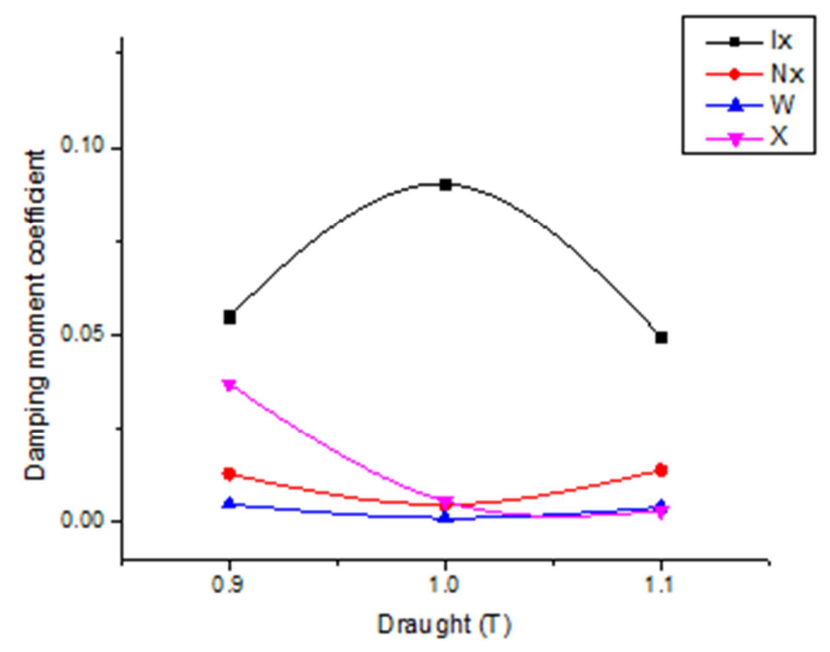

(a)

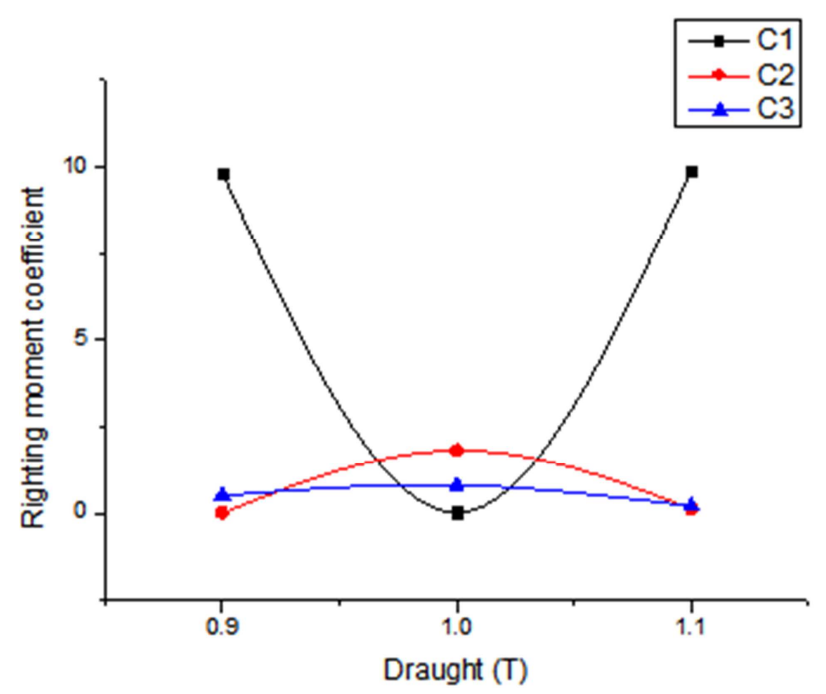

(b)

Figure 5. Curve of each moment coefficient with draught when the initial roll angle is $20^{\circ}$.
Through the identification calculation of the 9 sets of roll test data, the hull roll total moment of inertia Ix, the roll line type damping torque coefficient $\mathrm{Nx}$, the squared damping $\mathrm{W}$, the cubic damping $\mathrm{X}$ and the respective recovery moment coefficients are obtained in each case. The test shows the relationship between the hydrodynamic coefficients and the recovery moment coefficient under different initial roll angles under different draughts as shown in Figure 3-5:

It can be known from Figure 3-5 that the values of the torque coefficients will change with the change of the draft and the initial roll angle. When the initial roll angle is a small angle, the total hull of the hull is increased as the draft increases. When the roll angle is greater than $15^{\circ}$, the total moment of inertia decreases with the increase of draught. The possible reason is that when the draft is large, the wet area increases, the water resistance increases, and the roll angular velocity decreases, causing the roll the moment of inertia is reduced. When the angle is small, the linear damping coefficient, square damping and cubic damping moment increase first and then decrease with the increase of draught. At large angles, the squared damping and the cubic damping torque do not change significantly with the increase in draught, and remain close to zero and remain stable. It shows that at a large angle of $10^{\circ}-20^{\circ}$, the ship model does not have linear damping and square damping in the rolling motion, and the cubic damping is small and stable. Under the same roll angle, with the increase of draught, the recovery torque of the cubic is greatly changed. When the angle is small, it increases first and then decreases. When the angle is large, it decreases first and then increases. Both the quadratic recovery torque and the linear recovery torque are almost gradual, and the change is not obvious. The linear recovery torque is slowly decreasing and its value is close to zero.

The reasons for the abnormalities appearing in the summary chart are as follows. First, the large-angle roll test was accompanied by more obvious pitching and large interference factors. Second, the measured data samples are small and cannot accurately reflect their true changes. The third is the influence of environmental factors such as airflow and water waves during the test.

\section{Response Surface Fitting}

\subsection{Principle Introduction}

Response surface approximation (RSM) is a comprehensive application of experimental design and mathematical statistics. It can be applied to optimization design, which is to solve the approximation function between target and design variables through reasonable experimental design method. The basic idea is to express an implicit function by approximating a polynomial with a well-defined form.

If the parameter or design variable is assumed to be an $\mathrm{n}$-dimensional vector, $x \in \mathrm{E}^{\mathrm{n}}$, is the independent variable of the function to be solved, exist $\mathrm{y}=\mathrm{y}(\mathrm{x})$. Although no precise relational expression can be found, as long as a parameter value or a design variable value is given, that is if a sample 
$x^{(j)}$ is taken, the corresponding $y^{(j)}=y\left(x^{(j)}\right)$ can always be obtained from the test value, which is a response value that corresponds to a parameter value or a design variable value. As long as there are enough test samples, the sample points can be used to determine the response value. That is, using the undetermined coefficient method to solve the approximation function of the function $\mathrm{y}=\mathrm{y}(\mathrm{x})$ :

$$
\tilde{y}=f(x)
$$

Since the functional relationship between the response and the variable is unknown, it is necessary to determine its form in advance. And choosing a good function will make the approximation more accurate. The selection requirements generally satisfy two aspects: (1) the mathematical expression should be as simple as possible under the premise that the real function can be described; (2) the function should be used with as few undetermined coefficients as possible to reduce the work of physical testing and numerical analysis. The amount. In general, the form of linear or quadratic polynomial is usually chosen, as follows:

Linear type

$$
\tilde{y}=\alpha_{0}+\sum_{j=1}^{n} \alpha_{j} x_{j}
$$

Quadratic form without cross terms

$$
\tilde{y}=\alpha_{0}+\sum_{j=1}^{n} \alpha_{j} x_{j}+\sum_{i=1}^{n} \sum_{j=i}^{n} \alpha_{i j} x_{i} x_{j}
$$

Cubic type without cross term

$$
\tilde{y}=\alpha_{0}+\sum_{j=1}^{n} \alpha_{j} x_{j}+\sum_{j=1}^{n} \alpha_{j j} x_{j}^{2}+\sum_{j=1}^{n} \alpha_{j j j} x_{j}^{3}
$$

Cubic type with cross term

$$
\begin{array}{r}
\tilde{y}=\alpha_{0}+\sum_{j=1}^{n} \alpha_{j} x_{j}+\sum_{i=j}^{n} \sum_{j=1}^{n} \alpha_{i j} x_{i} x_{j}+\sum_{j=1}^{n} \alpha_{j j} x_{j}^{2}+\sum_{j=1}^{n} \alpha_{j j j} x_{j}^{3} \\
+\sum_{j \neq i}^{n} \sum_{i=1}^{n} \alpha_{i i j} x_{i}^{2} x_{j}+\sum_{k=i+1}^{n} \sum_{i=j+1}^{n} \sum_{j=1}^{n} \alpha_{i j k} x_{i} x_{j} x_{k}
\end{array}
$$

The above equations can be written in a uniform form:

$$
\tilde{y}=\sum_{j=0}^{k-1} \alpha_{j} x_{j}
$$

Where: $\alpha_{j}$ is the undetermined coefficient, $\mathrm{k}$ is the number of undetermined coefficients.

In order to determine the coefficient $\alpha_{j}$, we need to do m times ( $m \geq k$ ) independent test, and construct the response surface matrix as follows:

$$
X B \approx Y
$$

Where: X - variable matrix; Y - system response matrix; B coefficient matrix;

The coefficient matrix is calculated as follows:

$$
B=\left(X^{T} X\right)^{-1} X^{T} Y
$$

The fitting accuracy of the response surface is expressed by the complex correlation coefficient $R^{2}$ and the modified complex correlation coefficient $R_{a}^{2}$. The equation is as follows:

$$
\begin{gathered}
R^{2}=1-\frac{S S_{E}}{S S_{T}} \quad R_{a}^{2}=1-\left(\frac{m-1}{m-k}\right) \frac{S S_{E}}{S S_{T}} \\
S S_{T}=\sum_{i=1}^{n}\left(y_{i}-\bar{y}\right)^{2} \quad S S_{E}=\sum_{i=1}^{n}\left(y_{i}-\hat{y}\right)^{2}
\end{gathered}
$$

Where: $S S_{T}$ - the sum of squares; $\bar{y}$ - the mean of the response values; $S S_{E}$ - the sum of squared errors; $\hat{y}$ - the response surface at the corresponding test point value; $y_{i}$ - the test value of each test point; $m$ - the number of test points; $\mathrm{k}$ - the number of unknown parameters in the response surface equation.

Among them, the value of the complex correlation coefficient R2 for evaluating the accuracy of the response surface fitting varies between 0 and 1 , and the larger the value, the better the fitting effect. The modified complex correlation coefficient $R_{a}^{2}$ takes into account the influence of the number of unknown parameters and is used to compare the degree of approximation of the number of different parameters in the response surface function.

According to the design principle of the response surface method, a set of response surface software including five functional forms is prepared. The initial roll angle $\theta$ and the draft $\mathrm{T}$ are selected as design variables, and the data is calculated by the roll recognition software, fitting the equations of the torque coefficients at small angles.

\subsection{Torque Coefficient Response Surface Construction}

The equations with better comprehensive identification effect are selected to fit the results of the SWATH-USV identification calculation, and the relationship between the torque coefficients and the initial roll angle and draught was obtained.

Transverse total moment of inertia response surface structure: The system identification calculation data is selected for polynomial response surface fitting. The fitting accuracy is shown in Table 2. 
Table 2. Accuracy of the fitting of the polynomial response surface by the total moment of inertia of the roll.

\begin{tabular}{lll}
\hline Response surface fitting form: & Complex correlation coefficient & Modified complex correlation coefficient \\
\hline Linear type & 0.1235 & 0.0358 \\
Quadratic form without cross terms & 0.4039 & 0.3443 \\
Quadratic form with cross terms & 0.7268 & 0.6995 \\
Cubic type without cross term & 0.7105 & 0.6815 \\
Cubic type with cross term & 0.8357 & 0.8193 \\
\hline
\end{tabular}

Comparing the multi-correlation coefficients of different polynomials, the larger the value, the better the fitting effect. Therefore, the cross-third-order response surface is selected to fit the total inertia moment response surface equation of the roll.

$$
\begin{aligned}
I_{x x}^{\prime}= & -0.1148-19.6765 \theta+3.9014 \mathrm{~T}+35.2112 \theta \mathrm{T}+13.0856 \theta^{2}-6.8922 \mathrm{~T}^{2} \\
& -57.8905 \theta^{3}+3.7418 \mathrm{~T}^{3}+28.1686 \theta^{2} T-24.4368 \theta \mathrm{T}^{2}
\end{aligned}
$$

It should be noted that the complex correlation coefficient and the modified complex correlation coefficient are small here because the initial data used for the fitting is limited. The error between the fitted value and the identification value is shown in the figure. It can be seen that the error is within $10 \%$, which proves that the fitting result is available.

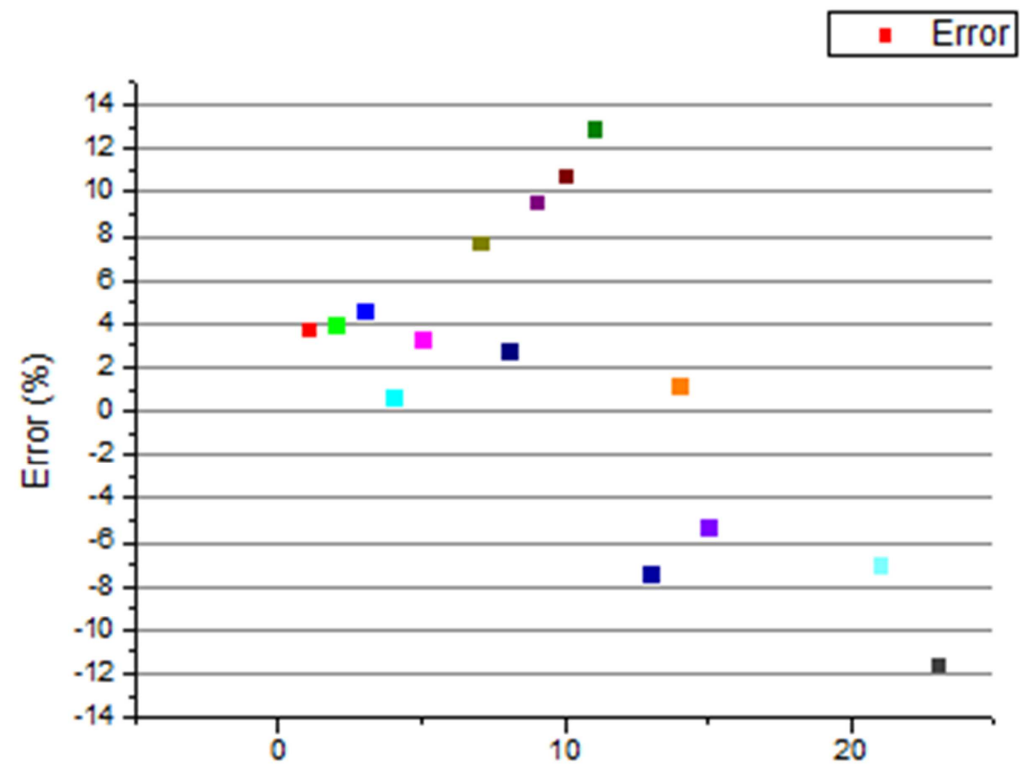

Figure 6. $I_{x x}^{\prime}$ Error between fitted value and identification value (\%).

Linear damping coefficient $\mathrm{N}$ response surface structure: The system identification calculation data is selected for polynomial response surface fitting. The fitting accuracy is shown in Table 3.

Table 3. Accuracy of linear damping coefficient $N$ fitting polynomial response surfacel.

\begin{tabular}{lll}
\hline Response surface fitting form: & Complex correlation coefficient & Modified complex correlation coefficient \\
\hline Linear type & 0.0165 & -0.0928 \\
Quadratic form without cross terms & 0.7117 & 0.6796 \\
Quadratic form with cross terms & 0.7475 & 0.7195 \\
Cubic type without cross term & 0.6712 & 0.6346 \\
Cubic type with cross term & 0.8672 & 0.8524 \\
\hline
\end{tabular}

The cross third-order response surface is selected to fit the linear damping coefficient response surface formula.

$$
\begin{aligned}
N= & 104.1235+331.4736 \theta-252.7236 T+126.3454 \theta T+1208.0589 \theta^{2} \\
& +246.0844 T^{2}-1685.8494 \theta^{3}-79.21104 T^{3}+15.1097 \theta^{2} T-68.6149 \theta T^{2}
\end{aligned}
$$

Square damping $\mathrm{W}$ response surface structure: Select the system identification calculation data for polynomial response surface fitting. The fitting accuracy is shown in Table 4. 
Table 4. Accuracy of nonlinear damping coefficient $W$ fitting polynomial response surface.

\begin{tabular}{lll}
\hline Response surface fitting form: & Complex correlation coefficient & Modified complex correlation coefficient \\
\hline Linear type & 0.2255 & 0.2195 \\
Quadratic form without cross terms & 0.2300 & 0.2239 \\
Quadratic form with cross terms & 0.2807 & 0.2751 \\
Cubic type without cross term & 0.3460 & 0.3409 \\
Cubic type with cross term & 0.3976 & 0.3929 \\
\hline
\end{tabular}

The cross third-order response surface is selected to fit the square damping coefficient W response surface formula.

$$
\begin{aligned}
W= & -0.0368+0.0335 \theta+0.9716 \mathrm{~T}-0.7373 \theta \mathrm{T}+0.0376 \theta^{2}-8.4418 \mathrm{~T}^{2} \\
& -0.0487 \theta^{3}+24.1735 T^{3}-0.1488 \theta^{2} T+3.7226 \theta T^{2}
\end{aligned}
$$

Cubic damping $\mathrm{x}$ response surface structure: The system identification calculation data is selected for polynomial response surface fitting. The fitting accuracy is shown in Table 5.

Table 5. Accuracy of the three-dimensional damping coefficient $x$ fitting polynomial response surface.

\begin{tabular}{lll}
\hline Response surface fitting form: & Complex correlation coefficient & Modified complex correlation coefficient \\
\hline Linear type & 0.0703 & -0.0226 \\
Quadratic form without cross terms & 0.3766 & 0.3142 \\
Quadratic form with cross terms & 0.5483 & 0.5031 \\
Cubic type without cross term & 0.1021 & 0.0123 \\
Cubic type with cross term & 0.7890 & 0.7679 \\
\hline
\end{tabular}

The cross third-order response surface is selected to fit the cubic square damping coefficient x response surface formula.

$$
\begin{aligned}
x= & -57.0832+186.0721 \theta+129.7801 \mathrm{~T}-87.4451 \theta \mathrm{T}-624.9291 \theta^{2}-116.9684 \mathrm{~T}^{2} \\
& +864.4606 \theta^{3}+34.6204 \mathrm{~T}^{3}-10.2646 \theta^{2} T+45.4604 \theta \mathrm{T}^{2}
\end{aligned}
$$

Nonlinear Restoration Torque Coefficient C1 Response Surface Structure: The system identification calculation data is selected for polynomial response surface fitting. The fitting accuracy is shown in Table 6.

Table 6. Accuracy of nonlinear restoring moment coefficient C1 fitting polynomial response surface.

\begin{tabular}{lll}
\hline Response surface fitting form: & Complex correlation coefficient & Modified complex correlation coefficient \\
\hline Linear type & 0.0426 & -0.0311 \\
Quadratic form without cross terms & 0.1000 & 0.0308 \\
Quadratic form with cross terms & 0.3200 & 0.2677 \\
Cubic type without cross term & -1.697 & -1.9040 \\
Cubic type with cross term & 0.4814 & 0.4415 \\
\hline
\end{tabular}

The cross third-order response surface is selected to fit the nonlinear restoration moment coefficient $\mathrm{C} 1$ response surface formula.

Nonlinear Restoration Torque Coefficient C2 Response Surface Structure: The system identification calculation data is selected for polynomial response surface fitting. The fitting accuracy is shown in Table 7.

$$
\begin{aligned}
C_{1}= & -1178.4651+6724.3109 \theta+2135.0278 \mathrm{~T}-8466.0770 \theta \mathrm{T}-11627.4743 \theta^{2}-1176.1008 \mathrm{~T}^{2} \\
& +20343.2172 \theta^{3}-0.0001 T^{3}-3387.2212 \theta^{2} T+5135.5859 \theta T^{2}
\end{aligned}
$$

Table 7. Accuracy of nonlinear restoration moment coefficient C2 fitting polynomial response surface.

\begin{tabular}{lll}
\hline Response surface fitting form: & Complex correlation coefficient & Modified complex correlation coefficient \\
\hline Linear type & 0.0762 & 0.0051 \\
Quadratic form without cross terms & 0.5638 & 0.5303 \\
Quadratic form with cross terms & 0.5638 & 0.5303 \\
Cubic type without cross term & 0.1489 & 0.0835 \\
Cubic type with cross term & 0.5918 & 0.5603 \\
\hline
\end{tabular}

The cross third-order response surface is selected to fit the nonlinear recovery moment coefficient $\mathrm{C} 2$ response surface formula. 


$$
\begin{aligned}
C_{2}= & -51.4098+13.9133 \theta+86.5451 \mathrm{~T}+184.9849 \theta \mathrm{T}-478.6870 \theta^{2}-36.5394 \mathrm{~T}^{2} \\
& +245.6646 \theta^{3}-3992499.276 \mathrm{~T}^{3}-1.1925 \theta^{2} T-167.3209 \theta \mathrm{T}^{2}
\end{aligned}
$$

Linear Restoration Torque Coefficient C3 Response Surface Structure: The system identification calculation data is selected for polynomial response surface fitting. The fitting accuracy is shown in Table 8 .

Table 8. Linear Restoration Torque Coefficient C3 Fitting Polynomial Response Surface Accuracy.

\begin{tabular}{lll}
\hline Response surface fitting form: & Complex correlation coefficient & Modified complex correlation coefficient \\
\hline Linear type & 0.2178 & 0.1576 \\
Quadratic form without cross terms & 0.3234 & 0.2713 \\
Quadratic form with cross terms & 0.4088 & 0.3633 \\
Cubic type without cross term & -0.6979 & -0.8285 \\
Cubic type with cross term & 0.4965 & 0.4578 \\
\hline
\end{tabular}

The cross third-order response surface is selected to fit the linear restoration moment coefficient $\mathrm{C} 3$ response surface formula.

$$
\begin{aligned}
C_{3}= & 75.0444-332.9668 \theta-133.2857 \mathrm{~T}+362.9278 \theta \mathrm{T}+693.4195 \theta^{2}+73.0928 \mathrm{~T}^{2} \\
& -1290.4322 \theta^{3}+7.7627 T^{3}+264.4304 \theta^{2} T-248.2497 \theta T^{2}
\end{aligned}
$$

\section{Conclusion}

In this paper, the rolling motion mode of the boat was studied by the static water roll test of the SWATH-USV and the system identification method. The mathematical model of hydrostatic roll, which was most suitable for the ship model under the nine working conditions, was found by the identification program. It can accurately describe the roll motion process and give the exact value of each torque coefficient, finding the variation law of each moment coefficient with the draft and roll angle. Using the response surface method, the relationship between each moment coefficient and the initial roll angle and draught change was fitted according to the calculation result of the best identification equation. The accuracy of the fitting equation was analyzed and the applicability was found. At the same time, the correctness of the system identification software was verified. The research shows that the system identification software can predict the future navigation parameters and motion posture of the SWATH-USV, and provide reference for further research on the rolling motion of the SWATH-USV.

\section{Acknowledgements}

This project is funded by the following projects: "National Natural Science Foundation", project approval number: 51379094; "Jiangsu Provincial Graduate Research and Practice Innovation Program Project", project approval number: KYCX17_1853.

\section{References}

[1] Ding Yong; Hu Kaiye; Qiu Minzhi. Lyapunov Characteristic Index Method for Nonlinear Rolling Motion Analysis of Ships [J]; China Shipbuilding; 2008.
[2] Zhang Zeguo;Yin Jianchuan;Hu Jiangqiang;Liu Cheng. Analysis of ship roll motion prediction based on grey model particle swarm optimization algorithm based on adaptive neural fuzzy inference system model[J];Science and Technology Engineering; 2016.

[3] Ma Lei;Zhang Xianku. Study on ship parameter excitation roll motion and its fin stabilizer control based on chaotic analysis [J]; Journal of Ship Mechanics; 2013.

[4] Li Hui; Guo Bichen; Li Xiaofang. Analysis and Prediction of Ship Rolling Motion Based on Wavelet Transform Theory [J]. Journal of Dalian Maritime University; 2010.

[5] Li Peiyong, Feng Tiecheng, Pei Yongming. Triangular ship rolling motion $[\mathrm{J}]$. China Shipbuilding, 2003.

[6] Li Hongxia, Lu Jiang, Gu Min, et al. Numerical simulation and analytical analysis of the strong excitation roll motion of the slant wave [J]. Chinese Ship, 2015.

[7] Liu Hongmei; Stability analysis and overturning probability calculation of ship rolling motion [D]; Tianjin University; 2004.

[8] Liu Cunjie. Study on the wave resistance and longitudinal motion stability of a SWATH-USV [D]. Jiangsu University of Science and Technology, 2016.

[9] Zhao Wei. Research on ship shape optimization and motion performance of SWATH-USV [D]. Harbin Engineering University, 2016.

[10] Li Guoying, Zhu Liangsheng, Ji Dongfang et al. Statistical Characteristics of Ship Rolling Motion Based on CFD Analysis [J]. Ship Science and Technology, 2012, 34(5): 18-22.

[11] Ma Xuequan, Ji Sheng, Wen Yiyan et al. Simulation of Ship Rolling Motion Mode Identification [J]. Journal of Shanghai Institute of Shipping Science, 2016, 39(2): 1-4. 